\title{
Structure and Magnetic Properties of Composite Toroids Powder Casted
}

\author{
MARCIN NABIALEK ${ }^{1}$, KATARZYNA BLOCH ${ }^{1}$, MICHAL SZOTA ${ }^{2}$, ANDREI VICTOR SANDU ${ }^{3,4 *}$ \\ ${ }^{1}$ Institute of Physics, Faculty of Production Engineering and Materials Technology, Czestochowa University of Technology, 19 \\ Armii Krajowej Str., 42-200 Czestochowa, Poland \\ ${ }^{2}$ Institute of Material Science and Engineering, Faculty of Production Engineering and Materials Technology, Czestochowa \\ University of Technology, 19 Armii Krajowej Str., 42-200 Czêstochowa, Poland \\ ${ }^{3}$ Gheorghe Asachi Technical University of lasi, Faculty of Materials Science and Engineering, 41 D. Mangeron Blvd., 700050, lasi, \\ Romania \\ ${ }^{4}$ Romanian Inventors Forum, 3 Sf. P. Movila Str., 700089, lasi, Romania
}

\begin{abstract}
The paper presents the results of research for magnetic composites made of metallic filler from alloy $\mathrm{Fe}_{65} \mathrm{CO}_{1} \mathrm{Ni}_{3} \mathrm{~W}_{2} \mathrm{~B}_{2}$ and epoxy resin EPIDIAN 100. The composites studied contained less than $10 \%$ of the resin, making them dimagnetoelectrics. The structure of alloys and composites was investigated using Xray diffraction and scanning electron microscopy and their magnetic properties using a vibration magnetometer. It has been shown that the structure and properties of the composites depend on the chemical composition of the metallic filler. And good link between components, with the proper placement of the filler in the matrix, has an impact on the quality of the composite.
\end{abstract}

Keywords: magnetic cores, composite, magnetic losses, mealing proces

Continuous civilization development fosters the emergence of ever newer materials to be used in highperformance devices. This means that such materials have better characteristics than their predecessors. A very intensively developing group of materials are composite materials that are used in all industries. In literature there are references to composites from a few thousand years ago but in fact their turbulent development began in the $40 \mathrm{~s}$ of the twentieth century when composites began to use all kinds metals of plastics [1,2]. There are three types of composites: layered, fibrous [2] and powder. One of the main advantages of composites is their ability to be molded freely, which is used inter alia in the production of magnetic cores. Such cores are made from a combination of various resin binder with a metallic filler. The filler may be a crystalline powder, partly crystallized or have amorphous structure. Crystalline materials have been widely used in the electrical industry for several decades. Production of crystalline textured transformer sheets is time consuming and expensive. These materials have quite a large disadvantage in the form of large magnetostriction, which is the reason for their loud work and overheating. For materials partially crystallized with grains of a nanometric dimension and in particular for amorphous materials the magnetostriction effect is near zero [3-5].

Therefore, these types of electrotechnical alloys are currently being intensively investigated for use in electrical engineering [6-8]. Unfortunately, their ability to form is limited. It is difficult to produce a ready-made amorphous or nanocrystalline core $[9,10]$. The reason for this fact is the crystallization itself, which takes place through diffusion processes at a sufficiently long time. Limiting the melt solidification time leading to an amorphous or nanocrystalline structure is directly related to the thickness of the obtained product, which is insufficient to produce the magnetic core. Therefore magnetic cores from amorphous or nanocrystalline alloys are obtained from powders mixed with a suitably selected binder. The properties of such composites depend on the size of the metallic particles their share and composition and on the binder itself. The filler's share determines the name of the magnetic material itself and its applicability. For a share of less than $1 \%$ of the diamagnetics (non-magnetic binders), such materials are called dimagnetoelectrics and for the share of 1 to $10 \%$ - magnetodielectrics. Particular use is given to magnetodielctrics which are low electrical permeability electrical insulators. However, not always the permeability of composites with resin content from 1 to $10 \%$ is low, what then? Can not this material be used? Composites with higher permeability than expected for magnetodielearics can be used as weakly magnetic baffles or wedges. Due to the rather low value of the coercion field, they can be used as magmatic screens. The structure of magnetic composites made of metal filler and epoxy resin can be simulated using the Finite Element Method. The research presented in this paper may provide the basis for endurance numerical analysis [11-19].

This paper presents the results of structural studies and properties of composites for use in power generation from powdered rapidly cooled alloys bonded using epoxy resin.

\section{Experimental part}

Materials and methods

Test specimens were made of pure ingredients: Fe $99.99 \%$ at, Ni $-99.999 \%$ at, Co $-99.98 \%$ at, W $-99.9999 \%$. Boron was added as an alloy FeB. All composites were prepared on the basis $\mathrm{Fe}_{65} \mathrm{CO}_{10} \mathrm{Ni}_{3} \mathrm{~W}_{2} \mathrm{~B}_{20}$ alloy.

Composite pellets were prepared in three stages. The first was the production of alloy ingots. Ingots were obtained by melting the alloy components in an arc furnace in Ar protection. Such smelters were divided into smaller batches for further production of composites. Rapidly cooled plates of $0.5 \mathrm{~mm}$ thickness and a surface of $10 \mathrm{~mm}$ $\mathrm{x} 10 \mathrm{~mm}$ were produced by the injection of liquid melt into the copper water cooled mold. This stage of composite production also took place in protective gas atmosphere. The produced plates were crushed and analyzed using X-

\footnotetext{
*email:sav@tuiasi.ro
} 
ray diffraction (BRUKER ADVANCE D8 CuKá). Samples were exposed within the $2 \theta$ angle range from $30^{\circ}$ to $100^{\circ}$ with resolution $0.02^{\circ}$ and exposure time to the measuring step $7 \mathrm{~s}$. Shredding of samples was done using a chilledchamber knife mill and separation into fractions using a set of sieves. Then a suitable portion of the metallic filler was weighed and dried dry with EPIDIAN 100 resin. After the mixing process, the solvent was added and the components of the composite remixed. The mixture thus prepared was pressed into pellets of $\sim 2 \mathrm{~mm}$ and $5 \mathrm{~mm}$ in diameter by means of a single-axis single-acting hydraulic press. The ironing time was $30 \mathrm{~s}$ and the force was $5 \mathrm{MPa}$. The discs were homogenized at $433 \mathrm{~K}$ and $90 \mathrm{~min}$. The $\mathrm{X}$ ray diffraction pattern was re-examined for such obtained samples. The crystalline phases present in the sample volume were determined on the basis of the analysis using the COD database. The surface profile of the pellets was analyzed using a $1.5 \mathrm{~mm}$ HOMMEL T1000 Tester. The surface of the samples was further examined by means of an electronic scanning microscope (Supra 35 ZEISS JOELJ SM 6610LV). The maximum operating voltage of the microscope was $20 \mathrm{kV}$, and the magnification was 10,000 times. Magnetic properties were determined by analyzing static magnetic hysteresis loops obtained from vibration magnetometer (VSM 7301 LAKE SHORE) measurements.
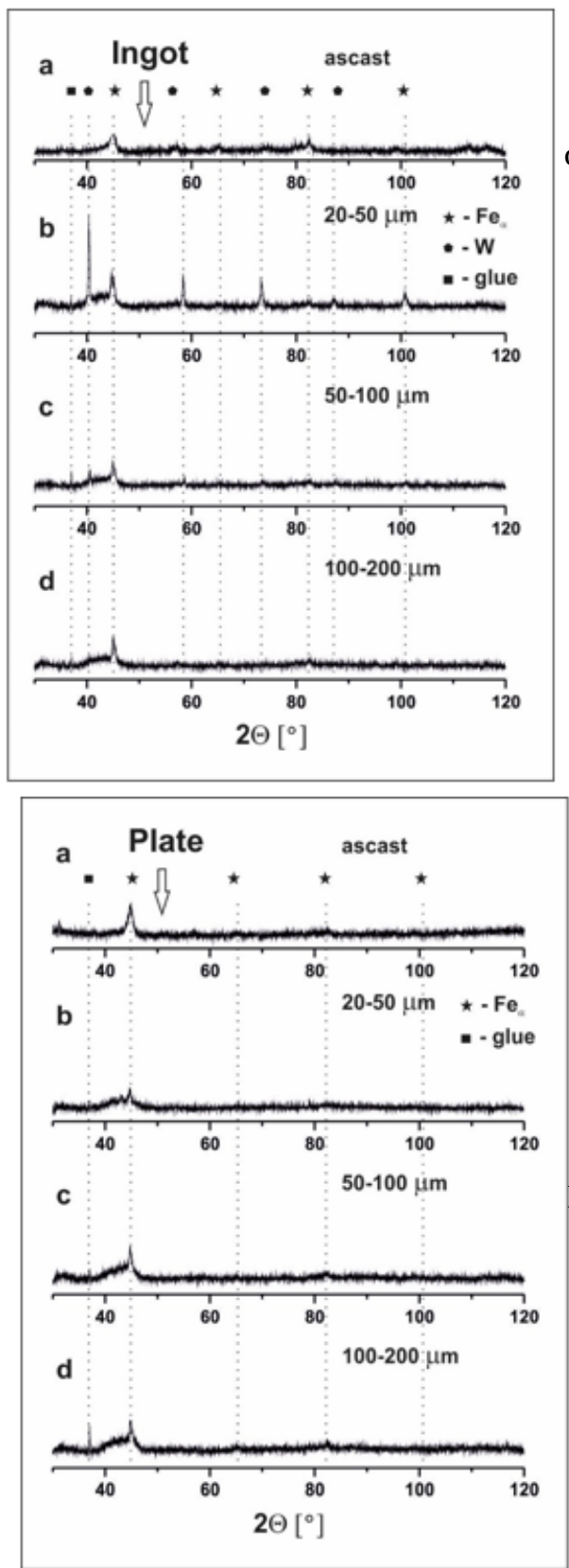

diffraction patterns obtained for the samples of the investigated alloy by slow solidification (a) and for the composite-based composite materials with the filler fraction: b $20-50 \mu \mathrm{m}$, c $50-100 \mu \mathrm{m}$ and d $100-200 \mu \mathrm{m}$

Fig. 2. X-ray diffraction patterns obtained for the samples of the alloy obtained in the rapid cooling process (a) and for the composite based on the filler metal fractions: b) $20-50 \mu \mathrm{m}$, c) $50-100 \mu \mathrm{m}$ and d) $100-200 \mu \mathrm{m}$

\section{Results and discussions}

Figure 1 shows patterns of X-ray diffraction of the sample in the form of a crystalline ingot and for obtained composites.

For samples in the form of a crystalline ingot and a composite formed on a metal filler of fraction $100-200$ $\mu \mathrm{m}$ the presence of one crystalline phase was identified: $\alpha-F e$. The shape of the peaks and their intensity suggest that the samples are nanomaterials with a low proportion of crystalline phase, which is estimated at several percent. In the case of composites made from powders of fractions: $20-50 \mu \mathrm{m}$ and $50-100 \mu \mathrm{m}$ in addition to the crystalline phase $\alpha$ - Fe the presence of tungsten diffraction reflections was observed. The shape of the tungsten diffraction peaks is similar to that of the $\alpha$ - Fe crystalline phase, which also suggests that the percentage of this phase in the volume of the sample is small, several percent. Figure 2 shows the images of X-ray diffraction for the fast-cooled plate and the composites produced after it has been crushed.

In the volume of samples: the fast-cooling plate and the composites produced therein, there were only crystal grains associated with the long-range ordering describing the phase $\alpha$ - Fe. Table 1 shows the results of X-ray diffraction analysis using the Scherrer equation. The calculated crystalline grains dimensions for the crystalline phase sample volume were not greater than $20 \mathrm{~nm}$.

Table 1

THE CRYSTALLINE GRAIN SIZE CALCULATED ON THE BASIS OF THE SCHERRER FORMULA FOR THE IDENTIFIED ORDERED LONG-RANGE $\alpha$ - Fe

\begin{tabular}{|l|c|c|}
\hline \multirow{2}{*}{} & \multicolumn{2}{|c|}{ Average size of crystalline grains [nm] } \\
\cline { 2 - 3 } & Ingot $\alpha$-Fe & Plate $\alpha-F e$ \\
\hline asmade & 8 & 8 \\
\hline $50-50 \mu \mathrm{m}$ & 13 & 13 \\
\hline $100-100 \mu \mathrm{m}$ & 12 & 15 \\
\hline
\end{tabular}

Figure 3 shows photographs taken using scanning electron microscope for the surface of the test specimens after the manufacturing process.

The analysis of the image shown in the picture (fig. 3) gives an opportunity to answer why only the sample cooled on a copper plate in addition to the crystalline phase $\alpha-\mathrm{Fe}$ tungsten reflections were observed and only in composites of fractions: $50-100 \mu \mathrm{m}$ and $100-200 \mu \mathrm{m}$. In the process of melting tungsten was not well melted (fig. 3a), large numbers of nearly $100 \mu \mathrm{m}$ unprocessed tungsten pieces

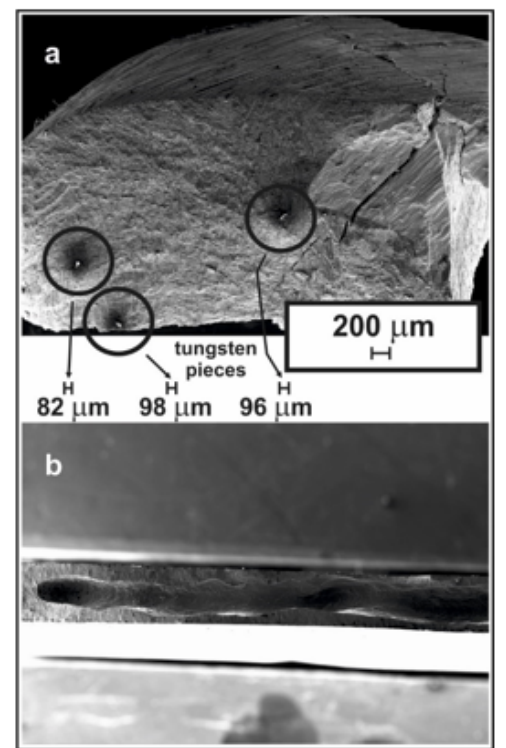

Fig. 3. Pictures of the breakthroughs of the samples in the solidified state: a - crystalline ingot, b - fast chilled plate 


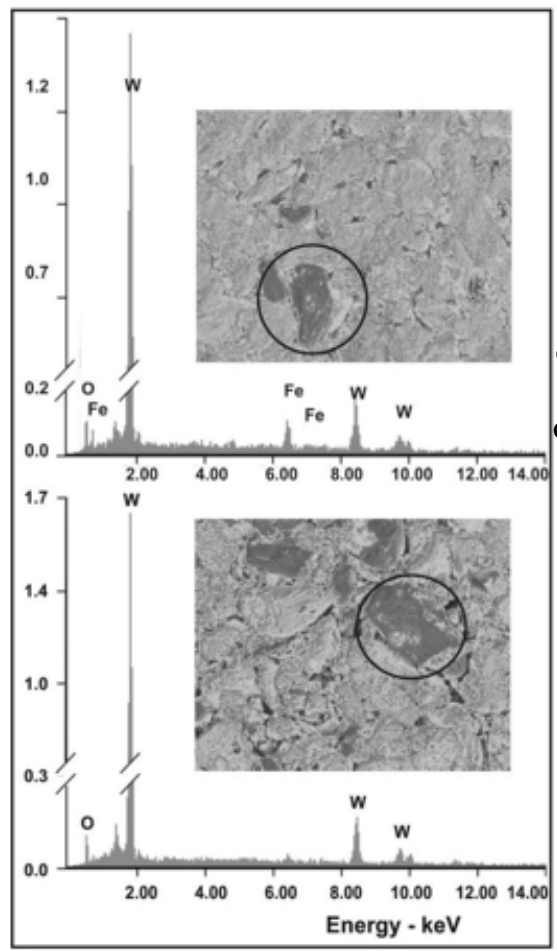

were marked with black circles. Unfortunately, the ability to estimate the average crystal particle size using the Schereer formula is limited by $100 \mathrm{~nm}$. Therefore, no such calculations were made, which is related to the analysis of SEM images. It was found that the additional melting of the crystalline ingot by the spin currents affected the homogenization of the alloy (fig. 3b). As described in section

Fig. 4. Analysis of chemical composition for areas marked by circles. Composite ( 50

- 100um fraction) produced from a cooled ingot on a copper plate details After being prepared, the samples were ground in a knife mill and then divided into fractions using a sieve set: 20 - $50 \mu \mathrm{m}, 50-100 \mu \mathrm{m}$ oraz $100-200 \mu \mathrm{m}$. This means that unprocessed tungsten pieces have been separated into fractions: 20 - $50 \mu \mathrm{m}$ and $50-100 \mu \mathrm{m}$ Because they had such a dimension (fig 3a). In addition, an analysis of the chemical composition of the particle composites (fig. 4) was performed. It has been shown to be pure tungsten.

Figure 5 shows static magnetic hysteresis loops obtained for solidified samples and composites formed on them. The solid specimens, both in the form of a cold rolled copper plate and a fast-cooled plate, had a high saturation magnetization of over $1.75 \mathrm{~T}$. Unfortunately, the value of the coercive field for samples in the post-manufacturing state was several thousand $\mathrm{A} / \mathrm{m}$, which disqualified them as material for use in magnetic cores of transformers. However, rapid cooling has been able to reduce the coil field by half from $6500 \mathrm{~A} / \mathrm{m}$ to $3200 \mathrm{~A} / \mathrm{m}$. An interesting correlation was found between coil field values and metal
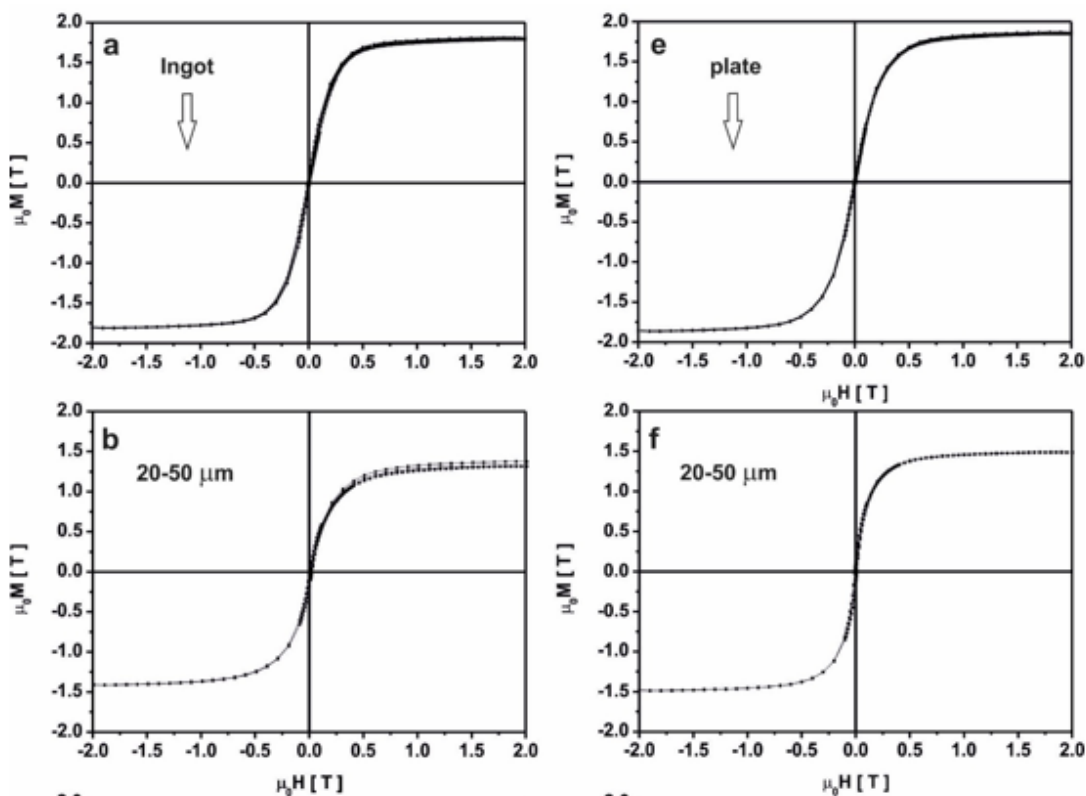

Fig. 5. Static magnetic hysteresis loops measured on samples in the state upon receipt $(a, e)$ and in the form of composites containing different metallic filler fractions $(b, c, d, f, g, h)$
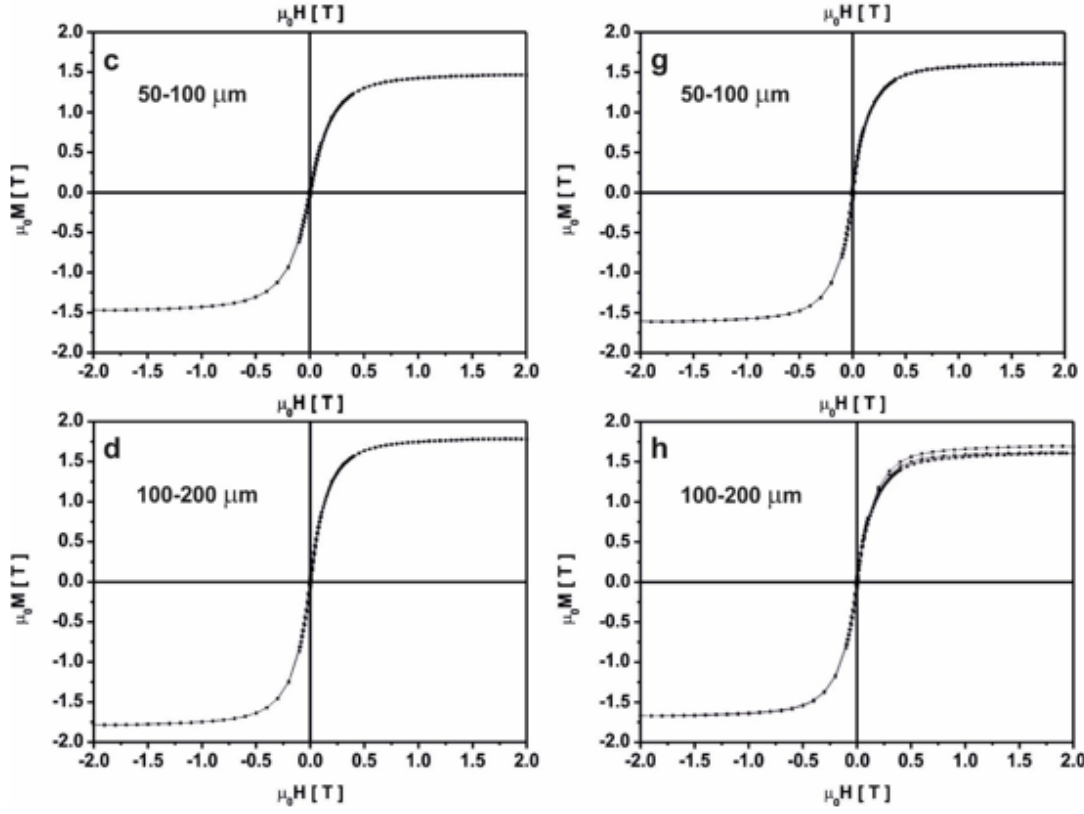


\begin{tabular}{|l|c|c|c|c|}
\hline \multirow{2}{*}{} & \multicolumn{2}{|c|}{ Ingot } & \multicolumn{2}{c|}{ Plate } \\
\cline { 2 - 5 } & Hc [A/m] & Hc [A/m] & Ms [T] & Hc [A/m] \\
\hline $20-50 \mu \mathrm{m}$ & 1.76 & 6715 & 1.85 & 3413 \\
\hline $50-100 \mu \mathrm{m}$ & 1.50 & 7024 & 1.48 & 6714 \\
\hline $100-200 \mu \mathrm{m}$ & 1.76 & 6190 & 1.60 & 6189 \\
\hline
\end{tabular}

Table 2

PARAMETERS OBTAINED FROM THE ANALYSIS OF STATIC MAGNETIC HYSTERESIS LOOPS: HC - FIELD OF COERCION, MS - MAGNETIC SATURATION filler fractions. For the composites produced, the value of the coercive field depended only on the metal filler fraction and not on the starting value as measured on the samples after solidification (table 2). On the other hand, the value of saturation magnetization increased with the size of the fraction $20-50 \mu \mathrm{m} \rightarrow 50-100 \mu \mathrm{m} \rightarrow 100-200 \mu \mathrm{m}$.

\section{Conclusions}

Using the fast cooling technique on a copper plate, samples of volatile alloys with small crystalline phase grains $\alpha$ - Fe. Unfortunately, an inaccurate melting process can cause melting alloy components like $W$ to melt not completely and occur in solidified melt in pieces of 20 to $100 \mu \mathrm{m}$. The presence of unmixed $\mathrm{W}$ significantly influences the increase in the coercivity field compared to the sample produced by the liquid melt aspiration method into the copper cooled mold. However, for the composite based composite fillers, the production process alone had no significant effect on the value of the coercive field. Evidently, the value of the coercive field depended on the amount of fraction used to produce the composite. The same was true for the magnetization of saturation, which also varied with the size of the metal filler fraction. It should also be noted that in the used knife mill to crush the samples, knife blades were made of tungsten. It was initially assumed that the tungsten composite was the result of a crushing of the mill's knives. However, the SEM study of ingots excluded this possibility. The Scherrer method gives the opportunity to estimate the average grain size that should be the same for the same material. Taking into account the shape of the X-ray diffraction patterns, where there are no narrow high intensity peaks, one can safely say that we are dealing with nanocrystalline material. The discrepancies of the determined crystalline grain dimensions are negligible and are related only to the accuracy of the half-widths of the peak and its maximum.

In conclusion, the studied alloyed compounds $\mathrm{Fe}_{65} \mathrm{CO}_{10} \mathrm{Ni}_{3} \mathrm{~W}_{2} \mathrm{~B}_{20}$ and $5 \%$ EPIDIAN epoxy resin are classified in the group of magnetodielectric materials. Without distinguishing between the cooling speed of the material, the composites have very similar utility parameters. The only drawback of the composites produced from the ingot was that too little melting of the alloying components was found, which only affected the properties of the alloys in the solidified state.

\section{References}

1.GAWDZINSK, K. Quality features of metal matrix composite castings, ARCHIVES OF METALLURGY AND MATERIALS, Volume: 58, Issue: 3, 2013, pp: 659-662, DOI: 10.2478/amm-2013-0051

2.PIESOWICZ E., IRSKA I., BRYLL K., GAWDZINSKA K., BRATYCHAK M., Poly (butylene terephthalate/carbon nanotubes nanocomposites, Part II. Structure and properties, Polimery 2016, $61 \mathrm{nr}$ 1, pp: 24-30, DOI: dx.doi.org/10.14314/polimery.2016.024

3.M. NABIALEK, J. All. Compd., 642, 2015, p. 98.

4.J. GONDRO, J.Magn. Magn. Mater., 632, 2017, p. 501.

5.K. BLOCH, M. NABIALEK, J. GONDRO, Physica B, 512, 2017, p. 81. 6.K. BLOCH, J. Magn. Magn. Mater., 390, 2015, p. 118.

7.K. BLOCH, M. NABIALEK, K. GRUSZKA, Rev. Chim. (Bucharest), 68, no. 4, 2017, p.698.

8.M. SZOTA, Arch. Metall. Mater, 62, 2017, p. 217.

9.D. SZEWIECZEK, S. LESZ, J Mater Process Tech 157, 2004, p.771.

10.S. LESZ, Mater. Character. 124, 2017, p. 97.

11.L.A. DOBRZANSKI, A. SLIWA, W. SITEK, Surf. Eng. 5 ISEC, 2006, p. 26.

12.A. SLIWA, B. BONEK, J. MIKULA, App. Sur. Sci. 388, 2016, p. 174.

13.A.SLIWA, W. KWASNY, W. SITEK, Arch. Metall. Mater.61, 2016, p. 481.

14.A. ZIELINSKI, M. SROKA, M. MICZKA, A. SLIWA, Arch. Metall. Mater.61, 2016, p. 753.

15.A. SLIWA, M.BONEK, Metalurgija, 56, 2017, p. 223.

16.M. NABIALEK, J. FUZER, L. DAKOVA, P. PIETRUSIEWICZ, Revi. Chim. (Bucharest), 68, no. 5, 2017, p. 1098.

17.M.B. PETRISOR, Crafting Global Competitive Economies: 2020 Vision Strategic Planning $\&$ Smart Implementation, Vols I-IV, 2014, p. 920.

18.M. POIANA, M. DOBROMIR, A.V. SANDU, V. GEORGESCU, Journal Of Superconductivity And Novel Magnetism, 25, 7, 2012, p. 2377.

19.S.I. TANASE, D. TANASE, A.V. SANDU, V. GEORGESCU, Journal of Superconductivity And Novel Magnetism, 25, 6, 2012, p. 2053

$\overline{\text { Manuscript received: } 14.01 .2017}$ 